\title{
A Noval Method for Detecting Plant Leaf Disease Using Image Processing and Deep Learning
}

\author{
M.Sowmiya ${ }^{a}$, S.Gnanapriya ${ }^{b}$, and P.M.Lavanya ${ }^{c}$ \\ $\mathbf{a}, \mathbf{b}, \mathbf{c}$ \\ Assistant Professor, Easwari Engineering College
}

Article History: Received: 11 January 2021; Accepted: 27 February 2021; Published online: 5 April 2021

\begin{abstract}
:
Abstract -The venture presents a programmed approach for early illness and nourishment insufficiency identification in plant leaf. A great many dollars are being spent to shield the harvests every year. Creepy crawlies, sustenance lack, plant illness and vermin harm the harvests and, in this way, are hazardous for the general development of the yield. One strategy to ensure the harvest is early illness identification and nourishment lack so the yield can be secured. The most ideal approach to think about the soundness of the yield is the convenient assessment of the harvest. On the off chance that sickness or sustenance inadequacy are identified, fitting measures can be taken to shield the harvest from a major creation misfortune toward the end. Early recognition would be useful for limiting the use of the pesticides and would give direction to the determination of the pesticides. It has become a wide territory for research now a days and a great deal of examination has been completed worldwide for programmed location of illnesses. Conventional technique for assessment of the fields is unaided eye assessment however it is exceptionally hard to have a point by point assessment in enormous fields. To inspect the entire field, numerous human specialists are required which is over the top expensive and tedious. Thus a programmed framework is required which can inspect the harvests to distinguish invasion as well as can characterize the kind of sickness on crops. PC vision procedures give viable approaches to breaking down the pictures of leaves. CNN is utilized for order of pictures with and without ailment dependent on the picture highlights. This procedure is less difficult when contrasted with the other mechanized strategies and gives better outcomes.
\end{abstract}

\section{Introduction}

In India, farming is the foundation of economy. Half of the populace is associated with cultivating exercises straightforwardly or in a roundabout way. Numerous assortments of organic products, oats and vegetables are created here and sent out to different nations. In plants, infections can be found in different parts, for example, natural products, stems and leaves. The principle infections of plants are viral, organism and bacterial illness like Alternaria, Anthracnose, bacterial spot, blister, and so forth.,. The viral sickness is because of ecological changes, growth illness is because of the nearness of parasite in the leaf and bacterial malady is because of essence of germs in leaf or plants. The proposed structure can be utilized to distinguish leaf sicknesses. Programmed identification of plant infections is a significant examination point since it can consequently recognize the ailments from the manifestations that show up on the plant leaves. Barbedo proposed a programmed technique for sickness manifestations division in advanced photos of plant leaves, in which shading channel control and Boolean activity are applied on paired cover of leaf pixels. He proposed the technique for self-loader division of plant leaf infection side effects in which the histograms of the $\mathrm{H}$ and shading channels are controlled. Ache et al proposed the technique for programmed division of yield leaf spot sickness pictures by incorporating neighborhood limit and cultivated locale developing. Singh and Misra proposed location of plant leaf ailments utilizing delicate processing strategies. Prasad et al proposed solo goal free based normal plant leaf infection division approach in which surface based grouping for division is finished. Du and Zhang proposed a method to fragment leaf picture with non-uniform brightening dependent on most extreme entropy and hereditary calculation (GA). Dhaygude and Kumbhar proposed rural plant leaf malady identification utilizing picture preparing in which the surface insights are figured from spatial dim level reliance grids (SGDM).

\section{Related Works}

There are a lot of leaf disease prediction systems out there for farmers and agriculturalists. Uday Pratap Singh [1] explains that the mango leaf disease can be identified using multilayer convolution neural network (MCNN).This can be used to differentiate between the healthy mango leaf and the fufus affected mango leaf.He insisted on using histogram of equalization on the realtime image of the leaf. David Blystone [2] in his thesis, has explained that a deep learning model that is highly accurate in identifying soyabean stresses from RGB images of soya bean leaves can be developed.This model behaves like black box predictor.This model is pulled off using deep CNN . Xi Yin [3] explains how multi modality imagery database can be used.MSU-PID is captured using the image sensors. They are fluorescence,infrared,RGB color and depth. 


\section{System Overview}

The system architecture diagram shown in Fig 1 consist of the image of the affected leaf. Then the image is pre processed and enhanced using grey scale and color scale conversion. The image is then segmented using edge detection and region based segmentation methods. Then feature extraction is use to extract the required data from the segmented image. Using $\mathrm{K}$ means clustering the objects will be grouped and then it will be compared with the disease database. This process efficiently identifies the Plant leaf disease.

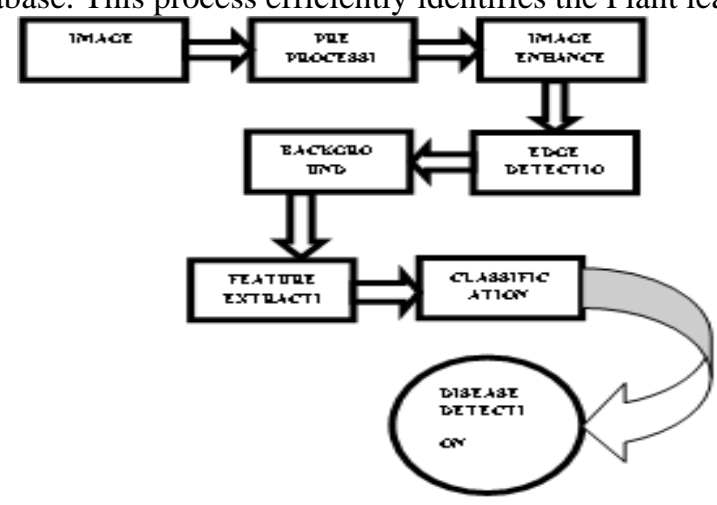

Fig 1: System Architecture

\section{Proposed System}

The proposed system consists of 4 modules, which are:

A. IMAGE PREPROCESSING AND ENHANCEMENT:

Image pre preparing is executed for improving the nature of the caught image. Image resizing, sifting, shading space change and histogram balance are the cycles done in pre processing. Grey scale change is one of the technique utilized for the upgrade of the image. Grey scale images are unmistakable from the slightest bit Biapparent high contrast images. Grey scale images have numerous shades of dark in the middle. Grey scale images can be the consequence of estimating the power of light at every pixel as per a specific weighted mixes of frequencies. Utilizing grey scale transformation the spots in the influenced leaves can be distinguished. Each leaf ailment has a particular pore design. Shading space change is additionally utilized for efficient identification.

B. IMAGE SEGMENTATION:

In plant infection acknowledgment applications, division is twofold. The initial segment of the division is to dispense with the foundation of the ailment influenced leaf. The second some portion of the division is to separate between the sickness influenced tissues with the solid tissue. Division is completed utilizing these two ,

EDGE DETECTION: Edges are Identified to identify the discontinuity between the leaf and the background of the image.

REGION BASED: Pixels that are identified with an item are assembled. In each progression at any rate one pixel is identified with the district and is mulled over.

C. FEATURE EXTRACTION:

Highlight extraction incorporates mining of information from the separated image of the plant leaf which could empower exact portrayal of the peculiarity. Highlights that could be isolated are surface(esentialness, unpredictability,homogeneity, and relationship), shape, size and shading.Textural highlights can be removed utilizing factual estimates, for example, Local Binary Patterns (LBP), Gray Level Co-event Matrix (GLCM), Color Cooccurrence Matrix (CCM) and Spatial Gray Level Dependence Matrix (SGLDM). Textural highlights can likewise be removed utilizing model-based strategies, for example, Auto-Regressive (AR) and Markov Random Field (MRF) models.

\section{D.CLASSIFICATION:}

- Deep learning algorithms are given feature vectors and arranged to arrange incorporates related with each ailment to be recognized.The arranged count would then have the option to be used to see features from new pictures got from the field.Classification oversees planning a given data incorporate vector with one of the specific classes aced during training.Alex net - plan include 5 convolutional layers and three filly related layers. Alex net's focal points-

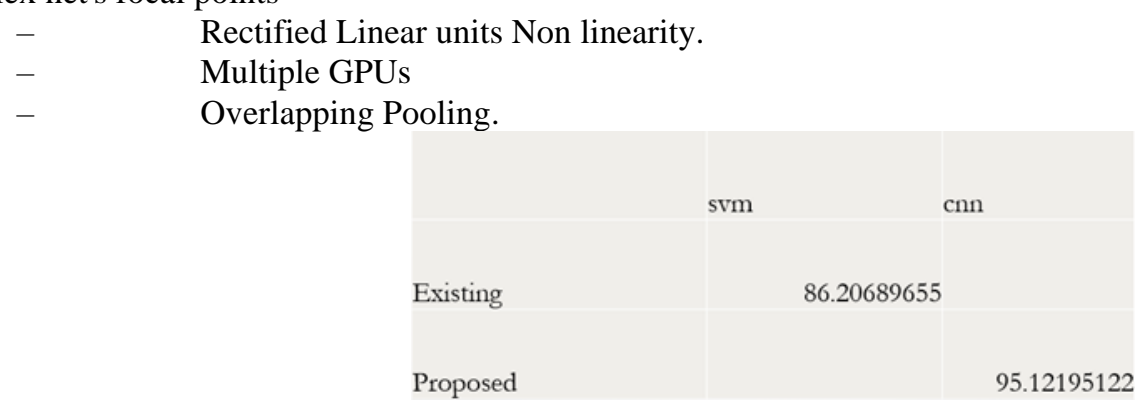

Fig 2: Performance Evaluation 


\section{Output Results}

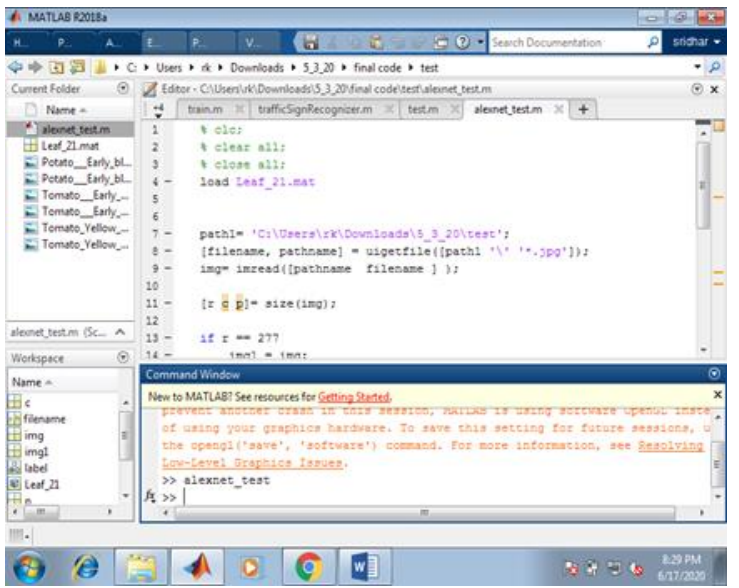

Fig 3 : Screenshot of the code

Fig 4 : Individual leaf selection

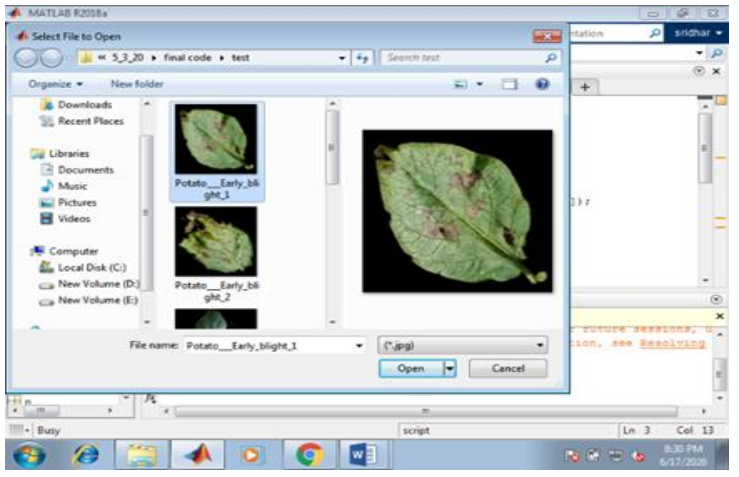

collected, Also the image enhancement techniques provided we predicts more disease detection ways than the current system handles. . The results can also be made available in all languages to give a better reach to more farmers across the country.

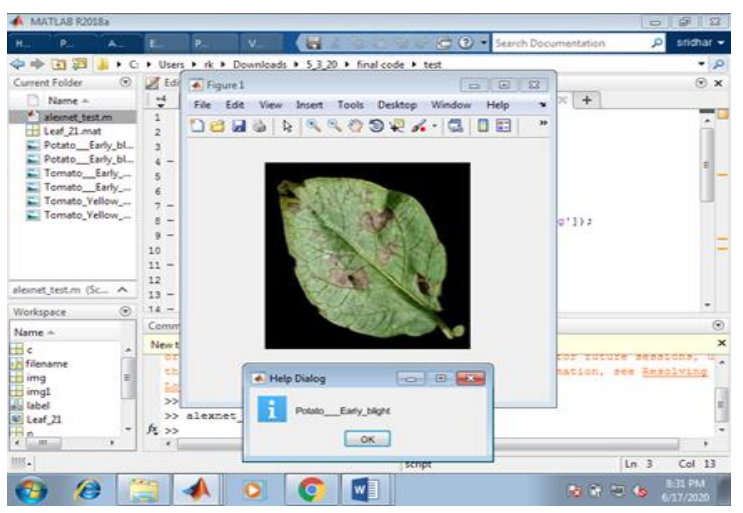

Fig 5 : Classified output for the selected leaf

\begin{tabular}{lcr} 
TOTAL SAMPLES $=100$ & \\
\multicolumn{1}{c}{ Exisiting } & Proposed \\
TP & 50 & 78 \\
FP & 8 & 4 \\
FN & 10 & 5 \\
TP=TRUE POSITIVE & & \\
FP=FALSE NEGATIVE & & \\
FN=FALSE NEGATIVE &
\end{tabular}

Fig 6 : comparing the collected samples with the existing system

\section{Future Works:}

Images from more sources can be obtained in order to analyse and predict the ways for disease detection. The pre processing of datasets can be changed for the enhancement of the image collected, Also the image 
enhancement techniques provided we predicts more disease detection ways than the current system handles. . The results can also be made available in all languages to give a better reach to more farmers across the country.

\section{Conclusion}

The proposed system aims at guiding the farmers by predicting the disease of the plant leaf so that quick action can be taken by the farmer to protect the plant from being destroyed. This system helps in the early detection of the plant leaf disease that will be very useful for the farmer to increase yield rather than being destroyed.

\section{References:}

1. Singh UP, Chouhan SS, Jain S, Jain S. Multilayer Convolution Neural Network for the Classification of Mango Leaves Infected by Anthracnose Disease. IEEE Access 2019.

2. Ghosal S,Blystone D, Singh AK, Ganapathysubramanian B, Singh A, Sarkar S. An explainable deep machine vision framework for plant stress phenotyping. Proc Natl Acad Sci U S A 2018.

3. Raj, T.N., Saranya, S., Murugan, S.A. and Bhuvaneswari, G., 2012. Secured multi path routing with trust establishment using mobile ad hoc networks. Int J Sci Eng Res, 3(1), pp.130-134.

4. Jeffrey A. Cruz · Xi Yin · Xiaoming Liu - Saif M. Imran3 - Daniel D. Morris · David M. Kramer· Jin Chen. Multi-modality imagery database for plant phenotyping 2018.

5. Too EC, Yujian L, Njuki S, Yingchun L. A comparative study of fine-tuning deep learning models for plant disease identification. Comput Electron Agric 2019.

6. Sampathkumar, A., Murugan, S., Elngar, A.A., Garg, L., Kanmani, R. and Malar, A.C.J., 2020. A Novel Scheme for an IoT-Based Weather Monitoring System Using a Wireless Sensor Network. In Integration of WSN and IoT for Smart Cities (pp. 181-191). Springer, Cham.

7. Picon A, Alvarez-Gila A, Seitz M, Ortiz-Barredo A, Echazarra J, Johannes A. Deep convolutional neural networks for mobile capture device-based crop disease classification in the wild. Comput Electron Agric 2019;161:280-90

8. Barbedo JGA. Impact of dataset size and variety on the effectiveness of deep learning and transfer learning for plant disease classification. Comput Electron Agric 2018;153:46-53.

9. S. K. S. Raja, R. Rishi, E. Sundaresan and V. Srijit, "Demand based crop recommender system for farmers," 2017 IEEE Technological Innovations in ICT for Agriculture and Rural Development (TIAR), Chennai, 2017, pp. 194-199, doi: 10.1109/TIAR.2017.8273714.

10. Barbedo JGA, Koenigkan LV, Halfeld-Vieira BA, Costa RV, Nechet KL, Godoy CV, et al. Annotated plant pathology databases for image-based detection and recognition of diseases. IEEE Lat Am Trans 2018.

11. Zhang X, Zhou X, Lin M, Sun J. ShuffleNet: An Extremely Efficient Convolutional Neural Network for Mobile Devices. Proc. IEEE Comput. Soc. Conf. Comput. Vis. Pattern Recognit., 2018.

12. Sampathkumar, A., Murugan, S., Rastogi, R., Mishra, M.K., Malathy, S. and Manikandan, R., 2020. Energy Efficient ACPI and JEHDO Mechanism for IoT Device Energy Management in Healthcare. In Internet of Things in Smart Technologies for Sustainable Urban Development (pp. 131-140). Springer, Cham. 